\title{
Übersichten
}

Somnologie $2020 \cdot 24: 106-114$ https://doi.org/10.1007/s11818-020-00238-9

Eingegangen: 3. Januar 2020

Angenommen: 22. Januar 2020

Online publiziert: 10 . Februar 2020

(c) Der/die Autor(en) 2020

Kai Spiegelhalder ${ }^{1}$ Jens Acker ${ }^{2} \cdot$ Harald Baumeister $^{3} \cdot$ Antje Büttner-Teleaga $^{4,5}$. Heidi Danker-Hopfe ${ }^{6}$. David D. Ebert ${ }^{7} \cdot$ Ingo Fietze $^{8}$. Lukas Frase $^{1}$. Sarah Klein ${ }^{9}$. Dirk Lehr $^{10} \cdot$ Andy Maun $^{11} \cdot$ Isabella Mertel ${ }^{12} \cdot$ Kneginja Richter $^{13} \cdot$ Dieter Riemann' . Cornelia Sauter $^{6} \cdot$ Claudia Schilling $^{14} \cdot$ Angelika A. Schlarb $^{15} \cdot$ Markus Specht $^{16} \cdot$ Lisa Steinmetz ${ }^{1} \cdot$ Hans G. Wee $\beta^{17} \cdot$ Tatjana Crönlein ${ }^{18}$

${ }^{1}$ Klinik für Psychiatrie und Psychotherapie, Universitätsklinikum Freiburg, Medizinische Fakultät, AlbertLudwigs-Universität Freiburg, Freiburg, Deutschland; ${ }^{2}$ Klinik für Schlafmedizin, Bad Zurzach, Schweiz; ${ }^{3}$ Abteilung für Klinische Psychologie und Psychotherapie, Universität Ulm, Ulm, Deutschland; ${ }^{4}$ Institut für Kognitive Wissenschaft, Woosuk Universität, Wanjugun, Südkorea; ${ }^{5}$ Fachbereich für Psychiatrie, Universität Witten/Herdecke, Witten, Deutschland; ${ }^{6}$ Kompetenzzentrum Schlafmedizin, Charité Universitätsmedizin Berlin, Berlin, Deutschland; ' ${ }^{7}$ Department of Clinical, Neuro- and Developmental Psychology, Vrije University Amsterdam, Amsterdam, Niederlande; ${ }^{8}$ Interdisziplinäres Schlafmedizinisches Zentrum, Charité - Universitätsmedizin Berlin, Berlin, Deutschland; ' 9 BARMER, Schwäbisch-Gmünd, Deutschland; ${ }^{10}$ Abteilung für Gesundheitspsychologie, Leuphana Universität Lüneburg, Lüneburg, Deutschland; " Lehrbereich Allgemeinmedizin, Medizinische Fakultät, Universitätsklinikum Freiburg, Freiburg, Deutschland; ${ }^{12}$ Schön Klinik Roseneck, Prien am Chiemsee, Deutschland; ${ }^{13}$ Klinik für Psychiatrie und Psychotherapie, Klinikum Nürnberg Nord, Nürnberg, Deutschland; ${ }^{14}$ Zentralinstitut für Seelische Gesundheit, Klinik für Psychiatrie und Psychotherapie, Medizinische Fakultät Mannheim, Universität Heidelberg, Mannheim, Deutschland; ${ }^{15}$ Fakultät für Psychologie und Sportwissenschaft, Arbeitseinheit Klinische Psychologie und Psychotherapie des Kindesund Jugendalters, Universität Bielefeld, Bielefeld, Deutschland; ${ }^{16}$ Zentrum für interdisziplinäre Schlafmedizin, DKD HELIOS Klinik Wiesbaden, Wiesbaden, Deutschland; ${ }^{17}$ Schlafzentrum am Pfalzklinikum Klingenmünster, Klingenmünster, Deutschland; ${ }^{18}$ Klinik für Psychiatrie und Psychotherapie, Universität Regensburg, Regensburg, Deutschland

\section{Digitale Behandlungsangebote für Insomnie - eine Übersichtsarbeit}

\section{Versorgungsdefizit und Fehlversorgung}

Ein- und/oder Durchschlafstörungen mit assoziierter Beeinträchtigung der Leistungsfähigkeit oder Tagesbefindlichkeit werden als Insomnien bezeichnet. Liegt keine organische Ursache zur Erklärung der schlafbezogenen Auffälligkeiten vor, wird die Diagnose nichtorganische Insomnie (F 51.0) oder Einund Durchschlafstörungen (G 47.0) anhand der ICD-10 vergeben [55]. Bei einer Kategorisierung gemäß DSM-5 wird die Diagnose „insomnia disorder“ verwendet [1]. Insomnien zählen zu den häufigsten Erkrankungen, mit denen Ärzte in der klinischen Versorgung der Allgemeinbevölkerung konfrontiert sind. Sie betreffen in chronischer Form 5-10\% der erwachsenen Bevölkerung in westlichen Industrienationen [37, 43] und bis $\mathrm{zu} 20 \%$ der Patienten in Hausarztpraxen [47].
Es ist allerdings anzunehmen, dass die Insomnie insgesamt unterdiagnostiziert ist. Beispielsweise zeigen Daten des Gesundheitsreports 2019 der BARMER[26], dass im Jahr 2017 in Deutschland nur 1,6\% der Versicherten eine Insomnie-Diagnose erhielten, womit im Vergleich mit epidemiologischen Daten mindestens $70 \%$ der Patienten unerkannt blieben oder nicht als Insomnie kodiert wurden [43].

Darüber hinaus wird der Großteil der Patienten derzeit nicht störungsangemessen behandelt. Während Leitlinien eine störungsspezifische psychotherapeutische Behandlung der Insomnie bei Patienten im Erwachsenenalter empfehlen [39, 40], wird diese sehr selten angewendet. So erhielten laut dem Gesundheitsreport der BARMER im Jahr 2017 nur etwa $10 \%$ der Versicherten mit einer Insomnie-Diagnose irgendeine psychotherapeutische Behandlung für irgendeine evtl. auch komorbid vorliegende andere Erkrankung [26]. In Bezug auf die in Leitlinien explizit nicht für die Langzeitbehandlung empfohlene pharmakologische Behandlung der Insomnie erhielten im Jahr 2017 10\% der an einer Insomnie erkrankten erwachsenen Versicherten der BARMER die Benzodiazepin-Rezeptor-Agonisten Zolpidem oder Zopiclon und 14\% Benzodiazepine. Dies ist sehr problematisch, da diese Substanzen bei längerer Einnahme zu Toleranz und Abhängigkeit führen und es insbesondere bei älteren Patienten, zu nächtlicher Verwirrtheit und Stürzen kommen kann [15]. Darüber hinaus erhielten etwa $9 \%$ der an einer Insomnie erkrankten Versicherten der BARMER Mirtazapin und 5\% Trimipramin, die in den Leitlinien ebenfalls nicht für die Langzeitbehandlung empfohlen werden, 
da zu ihrer Effektivität bei Insomnien kaum wissenschaftliche Evidenz vorliegt $[39,40]$.

Das beschriebene Versorgungsdefizit und die Fehlversorgung von InsomniePatienten sind gesundheitsökonomisch sehr relevant. So sind Insomnien vor allem aufgrund von verminderter Leistungsfähigkeit am Arbeitsplatz mit sehr hohen indirekten Gesundheitskosten verbunden. Diese betragen in Deutschland etwa $7800 €$ pro Patient pro Jahr, was jährliche Gesamtkosten von um die 40 Mrd. € ergibt. Davon würden sich laut einer vor drei Jahren veröffentlichten Studie mindestens $7 \mathrm{Mrd}$ € durch eine fachgerechte Behandlung einsparen lassen [50].

Die in den letzten Jahren veröffentlichten Leitlinien der Deutschen Gesellschaft für Schlafforschung und Schlafmedizin (DGSM) [40], der European Sleep Research Society (ESRS) [39] sowie des American College of Physicians (ACP) [38] empfehlen für das Erwachsenenalter die kognitive Verhaltenstherapie für Insomnien (KVT-I) als Behandlungsmethode der ersten Wahl für Insomnien. Diese auf den Schlaf fokussierte psychotherapeutische Kurzzeitbehandlung umfasst vier Module: 1) Psychoedukation zum Thema Schlaf; 2) Entspannungsübungen; 3) Bettzeitrestriktion und/oder Stimuluskontrolle; und 4) das Hinterfragen und Umstrukturieren dysfunktionaler Überzeugungen in Bezug auf das Thema Schlaf $[39,40]$. Vermutlich aufgrund fehlenden Wissens um die psychotherapeutischen Behandlungsoptionen für Insomnien, des höheren Zeitaufwands der KVT-I im Vergleich zu pharmakologischer Behandlung und eines Mangels an entsprechend ausgebildeten Therapeuten werden die Empfehlungen der Leitlinien jedoch bislang kaum und vor allem nicht rechtzeitig umgesetzt. Stattdessen kommt es zu der beschriebenen Überversorgung mit pharmakologischen Substanzen.

\section{Vorteile internetbasierter Therapieprogramme}

Ein vielversprechender Ausweg aus diesem Versorgungsdefizit für erwachsene Insomniepatienten könnte die Einfüh- rung internetbasierter Therapieprogramme basierend auf der evidenzbasierten KVT-I sein. Diese internetbasierten Therapieprogramme stehen den Patienten räumlich und zeitlich flexibel zur Verfügung, sodass auch Patienten in strukturschwachen Regionen erreicht werden können [18]. Zudem können die Patienten in einem selbst gewählten Tempo arbeiten und erforderliche Lernschritte so oft wie gewünscht wiederholen. Zusätzlich haben sie den Vorteil, dass sie frühzeitig im Krankheitsverlauf angewendet werden können, ohne lange Wartezeiten oder die Hemmschwelle, zum Arzt $\mathrm{zu}$ gehen. So werden auch vor allem die vielen Betroffenen mit einer beginnenden Insomnie erreicht. Die internetbasierten Therapieprogramme lassen sich damit auch in so genannte Stepped-CareModelle integrieren [19], indem sie in einer frühen Stufe der Versorgung niedrigschwellig zur Verfügung gestellt werden, bevor in späteren Stufen eine intensivere Behandlung stattfinden kann. Aktuelle Studien, die auf die Wirksamkeit von internetbasierten Therapieprogrammen hindeuten, werden im nachfolgenden Abschnitt zusammengefasst. Die hierfür durchgeführte Literaturrecherche basierte auf der Datenbank pubMed und folgendem Suchstring: (,internet“OR „web“) AND ,insomnia“. Zudem wurden die Literaturverzeichnisse der als relevant erachteten Artikel ebenfalls hinsichtlich möglicher weiterer relevanter Artikel durchgesehen. Die in die vorliegende Übersichtsarbeit eingeschlossenen Metaanalysen und Originalarbeiten $\mathrm{zu}$ digitalen Behandlungsangeboten für Insomnie sind in $\bullet$ Tab. 1 dargestellt.

\section{Effektivität internetbasierter KVT-I}

\section{Metaanalysen}

In einigen Metaanalysen wird eine gute Effektivität der internetbasierten Behandlung bei erwachsenen Insomniepatienten deutlich. In einer Metaanalyse von 11 Studien von Zachariae et al. [56] werden für die onlinebasierte KVT-I im Vergleich mit Warteliste (9 Studien), Treatment as usual (1 Studie) oder aktiver Kontrollbedingung (1 Studie) mittlere bis große Effektstärken (Hedges g) angegeben für die Schwere der insomnischen Symptomatik [1], für die subjektive Schlafeffizienz $(0,58)$, für die subjektive Schlafqualität $(0,49)$ und für die subjektive nächtliche Wachzeit nach dem Einschlafen $(0,45)$. Geringe Effektstärken wurden für die subjektive Einschlaflatenz $(0,41)$ und für die subjektive Schlafdauer $(0,29)$ angegeben, wobei die subjektiven Angaben in der Regel auf Schlaftagebuchdaten beruhen.

In der aktuellsten Metaanalyse, die neben der konventionellen „face-toface " (F2F) KVT-I auch internetbasierte Behandlungsprogramme einschloss, ergaben sich vergleichbare Werte [52]. So zeigten sich auch hier mittlere bis hohe Effektstärken (Hedges g) für die Schwere der insomnischen Symptomatik $(0,98)$, für die subjektive Schlafeffizienz $(0,71)$, für die subjektive Einschlaflatenz $(0,57)$, für die subjektive nächtliche Wachzeit nach dem Einschlafen $(0,63)$ und geringe Effektstärken für die subjektive Schlafqualität $(0,40)$ und für die subjektive Schlafdauer $(0,16)$. Die Metaanalyse von van Straten et al. [52] zeigte in Bezug auf die Schwere der insomnischen Symptomatik (nicht aber in Bezug auf die subjektive Schlafeffizienz und die subjektive Einschlaflatenz) Hinweise auf eine Überlegenheit der F2F-KVT-I im Einzelsetting gegenüber Selbsthilfeprogrammen einschließlich internetbasierter Programme ohne therapeutische Unterstützung.

\section{Randomisierte kontrollierte Studien}

In vielen Untersuchungen zur internetbasierten Behandlung der Insomnie wurden Wartelistenkontrollbedingungen als Vergleichsgruppe eingesetzt, u. a. in Ritterband et al. [41]. Es gibt aber auch Studien, die eine Wirksamkeit der Behandlung mit einer psychologischen PlaceboBehandlung [20] verglichen und die gute Wirksamkeit ebenfalls bestätigten.

Direkte Vergleichsstudien von internetbasierter KVT-I zu F2F-psychotherapeutischer Behandlung zeigten bislang keine ganz einheitlichen Ergebnisse. Während in einer Arbeit aus den Niederlanden die F2F-Behandlung ei- 
ner internetbasierten Behandlung mit Therapeutenunterstützung per E-Mail deutlich überlegen war [32], war diese Überlegenheit etwas geringer in einer Studie an US-Militärangehörigen ausgeprägt, in der eine F2F-Behandlung mit einer internetbasierten Behandlung ohne Therapeutenunterstützung verglichen wurde [48]. In einer anderen Arbeit zeigte sich eine Nicht-Unterlegenheit einer internetbasierten Behandlung mit Therapeutenunterstützung per E-Mail und Telefonkontakten mit einer Gruppenbehandlung im F2F-Format [7]. Kritisch anzumerken bei allen genannten Studien ist, dass diese für Nicht-Unterlegenheitsstudien eine nicht ausreichende statistische Power hatten und dass z.T. kein äquivalentes Ausbildungsniveau bezüglich der therapeutischen Kompetenzen zwischen den Therapeuten der beiden Bedingungen (F2F-Bedingung, internetbasierter Behandlung) vorlag [32]. Bei anderen psychischen Störungen und körperlichen Erkrankungen zeigt eine Metaanalyse über 20 RCTs zum direkten Vergleich von therapeutisch begleiteter internetbasierter KVT und F2F-KVT keinen Unterschied zwischen diesen beiden Ansätzen mit einer gepoolten Effektstärke zum Zeitpunkt nach Therapie von Hedges $g=0,05$ [10].

Grundsätzlich zeigte sich in den Originalstudien bei erwachsenen Insomniepatienten direkt im Anschluss an die durchgeführte Therapie eine gute Effektivität der Onlinebehandlung. Zu den Followup-Messzeitpunkten ergab sich darüber hinaus auch eine Stabilität der erzielten Behandlungserfolge bis zu 3 Jahre nach Beendigung der eigentlichen Therapie [8, $42,53]$. Die Studie von Ritterband et al. [42] zeigte beispielsweise auch in der Follow-up-Untersuchung nach einem Jahr noch große Effektstärken für die Schwere der insomnischen Symptomatik, die Einschlaflatenz und die nächtliche Wachzeit nach dem Einschlafen gegenüber Psychoedukation allein.

Es liegt gute Evidenz dafür vor, dass die internetbasierte KVT-I auch effektiv ist, Symptome von Depressivität, Angst und Fatigue zu reduzieren und die Lebensqualität der Studienteilnehmer zu verbessern. So zeigte sich beispielsweise in einer Untersuchung von Hagatun et al.
Somnologie $2020 \cdot 24: 106-114$ https://doi.org/10.1007/s11818-020-00238-9

(c) Der/die Autor(en) 2020

K. Spiegelhalder · J. Acker · H. Baumeister · A. Büttner-Teleaga · H. Danker-Hopfe · D. D. Ebert · I. Fietze - L. Frase - S. Klein · D. Lehr · A. Maun · I. Mertel · K. Richter · D. Riemann · C. Sauter · C. Schilling $\cdot$ A. A. Schlarb $\cdot$ M. Specht $\cdot$ L. Steinmetz $\cdot$ H. G. Weeß $\cdot$ T. Crönlein

\section{Digitale Behandlungsangebote für Insomnie - eine Übersichtsarbeit}

\begin{abstract}
Zusammenfassung
Digital angebotene psychologische Interventionen gegen Schlafstörungen sind aktuell ein sehr intensiv bearbeitetes Forschungsthema. In dieser Übersichtsarbeit werden Originalarbeiten und Metaanalysen zu diesem Thema zusammengefasst. Hierbei zeigt sich, dass die internetbasierte kognitive Verhaltenstherapie für Insomnie (KVT-I) bei Erwachsenen durchweg sehr effektiv ist mit allenfalls leicht geringeren Effektstärken als die gleiche Behandlung mit physischer Präsenz von Therapeuten und Patienten. Behandlungseffekte zeigen sich dabei auch für sekundäre Outcome-Parameter wie Depressivität, Angst, Fatigue und Lebens-
\end{abstract}

qualität. Hingegen lassen die Forschung zur Wirksamkeit der internetbasierten KVT-I bei Erwachsenen mit komorbiden psychischen Störungen oder körperlichen Erkrankungen sowie die Forschung zur Frage, wieviel Therapeutenkontakt in die Behandlung integriert werden sollte, anscheinend noch keine abschließenden Antworten zu. In diesen Bereichen scheint weitere Forschung notwendig zu sein scheint.

\section{Schlüsselwörter}

Ein- und Durchschlafstörungen · Kognitive Verhaltenstherapie · Internet · Fatigue . Depression

\section{Online insomnia treatment-a review}

\begin{abstract}
Digitally provided psychological interventions for sleep disorders are currently a very intensively researched topic. In this review, original work and meta-analyses are summarized. Thus, it was shown that internetbased cognitive behavioral therapy for insomnia (CBT-I) in adults is consistently very effective, with only slightly reduced effects compared with the same treatment with the physical presence of therapists and patients. Treatment effects are also shown for secondary outcome parameters such as depression, anxiety, fatigue, and quality
\end{abstract}

of life. On the other hand, research on the effectiveness of internet-based CGT-I in adults with comorbid mental disorders or physical illnesses, and on the issue of how much contact with therapists should be integrated into treatment, does not seem to provide any conclusive answers. Further research seems to be needed in these areas.

\section{Keywords}

Sleep initiation and maintenance disorders . Cognitive behavioral therapy - Internet .

Fatigue - Depression
[28], dass eine internetbasierte KVT-I ohne Therapeutenunterstützung $(n=95)$ im Vergleich mit einer Psychoedukationsbedingung $(n=86)$ zu einer signifikanten Reduktion von Ängstlichkeit, Depressivität und Fatigue führte, wobei mittlere bis große Effektstärken erzielt wurden. In Bezug auf Depressivität zeigten sich ähnliche Ergebnisse in einer Studie von Cheng et al. [12]. Darin wurden 658 erwachsene Patienten mit Insomnie randomisiert einer internetbasierten KVT-I $(n=358)$ bzw. einer psychoedukativen Kontrollbedingung $(n=300)$ zugewiesen. Es wurde ein stärkerer Effekt der digitalen KVT-I sowohl auf schlafbezogene Parameter als auch auf Depressivität gefunden. Dabei zeigte sich eine Effektstärke (Hedges g) von 0,64 für die Depressivität, was in Bezug auf die psychotherapeutische Veränderung von Depressivität ungewöhnlich hoch ist. Gosling et al. [27] untersuchten die Effekte einer internetbasierten Behandlung auf Ängstlichkeit und bestätigten die Ergebnisse von Hagatun et al. [28]. In einer weiteren großangelegten randomisierten klinischen Studie wurde der Effekt der internetbasierten KVT-I im Vergleich zu Psychoedukation auf die Lebensqualität von 1711 erwachsenen Patienten mit Insomnie untersucht 
Tab. 1 Metaanalysen und Originalarbeiten zu digitalen Behandlungsangeboten für Insomnie, die die Grundlage dieser Übersichtsarbeit darstellen

\section{Referenz}

Stichprobengröße

Ergebnisse

METAANALYSEN

Van Straten et al., 2018 [52]

6303 Vpn mit INS

Zachariae et al., 2016 [56] 1460 Vpn mit INS

\section{RANDOMISIERTE KONTROLLIERTE STUDIEN}

Blom et al., 2015 [6]

Blom et al., 2015 [7]

Blom et al., 2016 [8]

Blom et al., 2017 [9]

Cheng et al., 2019 [12]

Christensen et al., 2016

[14]

Ebert et al., 2015 [17]

Espie et al., 2012 [20]

Espie et al., 2019 [22]

Forsell et al., 2019 [23]

Glozier et al., 2019 [25]

Gosling et al., 2018 [27]

Hagatun et al., 2018 [28]

Lancee et al., 2013 [31]

Lancee et al., 2016 [32]

Lorenz et al., 2019 [33]

McGrath et al., 2017 [35]

Mindell et al., 2011 [36]

Ritterband et al., 2009 [41]

Ritterband et al., 2017 [42]

Schlarb et al., 2017 [44]

Shaffer et al., 2019 [46]

Taylor et al., 2017 [48]

Thiart et al., 2015 [49]

Thiart et al., 2016 [50]

Vedaa et al., 2019 [53]

Zachariae et al., 2018 [57]

ANDERE STUDIEN

Chan et al., 2017 [11]

Cheung et al., 2019 [13]

Dozeman et al., 2017 [16]

Luyster et al., 2018 [34]

Schlarb \& Brandhorst, 2012 [45]
43 Vpn mit INS und Depression

48 Vpn mit INS

148 Vpn mit INS

43 Vpn mit INS und Depression

658 Vpn mit INS

1149 Vpn mit INS und subklinischer Depression

128 Vpn mit INS

164 Vpn mit INS

1711 Vpn mit INS

251 Vpn mit INS

87 Vpn mit INS und Depression

1149 Vpn mit INS und subklinischer Depression

181 Vpn mit INS

262 Vpn mit INS

90 Vpn mit INS

56 Vpn mit INS

134 Vpn mit INS und Hypertonie

264 Kleinkinder mit INS (Alter: 6-36 Monate), mit Müttern

44 Vpn mit INS

303 Vpn mit INS

199 Kleinkinder mit INS (Alter: 6-63 Monate), mit Eltern

151 Vpn mit INS und Schmerzen

100 Vpn mit INS

$128 \mathrm{Vpn}$ mit INS

$128 \mathrm{Vpn}$ mit INS

$95 \mathrm{Vpn}$ mit INS

255 Vpn mit INS und Brustkrebs

10 Vpn mit INS

51 Vpn mit INS

171 Vpn mit INS und Brustkrebs

23 Vpn mit INS und Asthma

55 Kleinkinder mit INS (Alter: 8-57 Monate), mit Eltern
KVT-I ist sehr wirksam in der Behandlung der INS (z. B. $g=0,98$ in Bezug auf die Symptomschwere)

iKVT-I ist sehr wirksam in der Behandlung der INS (z. B. $g=1,09$ in Bezug auf die Symptomschwere)

iKVT-I ist wirksamer als iKVT für Depression bei Patienten mit INS und Depression iKVT-I ist ähnlich wirksam wie F2F-KVT-I (Gruppentherapie)

iKVT-I ist auch bei Follow-up nach drei Jahren noch sehr wirksam

iKVT-I ist auch bei Follow-up nach drei Jahren wirksamer als iKVT für Depression bei Patienten mit INS und Depression

iKVT-I ist wirksam in der Reduktion von Depressivität

iKVT-I ist wirksam in der Reduktion von Depressivität, ein präventiver Effekt auf inzidente Fälle von diagnostizierter Depression zeigte sich hingegen nicht

iKVT-I ist wirksam in der Behandlung der INS

iKVT-I ist wirksam in der Behandlung der INS

iKVT-I ist auch wirksam in der Verbesserung der Lebensqualität

Es ist sinnvoll, potenzielle Non-Responder auf iKVT-I früh zu erkennen und intensiver zu behandeln

iKVT-I ist wirksam in der Reduktion von Depressivität

iKVT-I ist wirksam in der Reduktion von Ängstlichkeit

iKVT-I ist wirksam in der Reduktion von Ängstlichkeit, Depressivität und Fatigue Therapeutenunterstützung durch E-Mails erhöht die Wirksamkeit von iKVT-I

F2F-KVT-I ist iKVT-I mit Therapeutenunterstützung überlegen

iKVT-I ist wirksam in der Behandlung der INS

iKVT-I ist nicht wirksam in der Reduktion des Blutdrucks

iKVT-I ist wirksam in der Behandlung der INS bei Kleinkindern

iKVT-I ist wirksam in der Behandlung der INS

iKVT-I ist wirksam in der Behandlung der INS, auch bei Follow-up nach 1 Jahr

iKVT-I ist wirksam in der Behandlung der INS bei Kleinkindern

iKVT-I ist wirksam in der Behandlung der INS bei komorbiden Schmerzen

iKVT-I ist wirksam in der Behandlung der INS

iKVT-I ist wirksam in der Behandlung der INS

iKVT-I ist kosteneffektiv

iKVT-I ist wirksam in der Behandlung der INS, auch bei Follow-up nach 18 Monaten

iKVT-I ist wirksam in der Behandlung der INS bei komorbidem Brustkrebs

Bettzeitrestriktion wird bei iKVT-I von einigen Vpn als problematisch erlebt

Betroffene haben verschiedene Präferenzen für iKVT-I oder F2F-KVT-I

Prä-zu-Post-Vergleiche suggerieren Effektivität von iKVT-I bei komorbidem Brustkrebs

Prä-zu-Post-Vergleiche suggerieren Effektivität von iKVT-I bei komorbidem Asthma

Prä-zu-Post-Vergleiche suggerieren Effektivität von iKVT-I in der Behandlung der INS bei Kleinkindern 
[22]. Dabei zeigten sich insbesondere große Effekte auf individuell erfasste Lebensbereiche, die durch Schlafstörungen besonders beeinträchtigt waren.

\section{Effektivität für spezifische Zielgruppen}

Die internetbasierte KVT-I wurde auch bei Patienten eingesetzt, die komorbid zur Insomnie noch weitere psychische oder körperliche Erkrankungen aufwiesen. Die größte Anzahl an Studien liegt dabei für Patienten vor, die unter einer depressiven Erkrankung litten.

\section{Internetbasierte KVT-I im Kontext von Depressionen}

Blom et al. [6] untersuchten in einer ersten randomisierten klinischen Studie 43 erwachsene Patienten mit Insomnie und diagnostizierter Depression und verglichen die internetbasierte KVT-I mit einer internetbasierten Behandlung für Depression, die ebenfalls auf den Prinzipien der kognitiven Verhaltenstherapie beruhte. Die internetbasierte KVT-I war der internetbasierten Depressionsbehandlung hierbei in Bezug auf die Verbesserung des Schlafs überlegen und in Bezug auf die Minderung von Depressivität gleichwertig. Die Effekte zu Therapieende blieben in der Follow-upUntersuchung nach drei Jahren stabil [9]. In einer weiteren Arbeit von Glozier et al. [25] wurden 87 Patienten mit Insomnie und diagnostizierter Depression randomisiert einer internetbasierten KVT-I oder einer internetbasierten psychoedukativen Kontrollbedingung zugewiesen. Während sich die insomnischen Beschwerden in dieser Studie stärker unter der internetbasierten KVT-I besserten, zeigte sich kein statistisch signifikanter Effekt für die Depressivität. In einer großangelegten randomisierten klinischen Studie wurde untersucht, inwieweit sich das Auftreten von Depressionen durch eine frühzeitige Behandlung von Schlafstörungen bei Erwachsenen verhindern lässt [14]. In dieser Studie wurden 1149 Patienten mit Insomnie und subklinischen Depressionssymptomen entweder einer internetbasierten KVT-I oder einer psychoedukativen Kontrollbedin- gung zugewiesen. Die internetbasierte KVT-I konnte depressive Symptome im Vergleich zur Kontrollbedingung signifikant verringern, jedoch nicht die Inzidenz von depressiven Erkrankungen, vermutlich auch da die Anzahl von neu aufgetretenen depressiven Erkrankungen im Beobachtungszeitraum sehr klein war $(n=22)$. Insgesamt ist die Evidenz zum Thema internetbasierte Behandlung der Insomnie im Kontext von Depression uneinheitlich, und es bedarf weiterer Forschung zu diesem wichtigen Themenkomplex.

\section{Internetbasierte KVT-I im Kontext somatischer Erkrankungen}

Darüber hinaus wurde die Effektivität der internetbasierten KVT-I bei Patientinnen mit Brustkrebs und insomnischen Symptomen untersucht. Sowohl eine unkontrollierte Pilotstudie [16] als auch eine randomisierte klinische Studie mit 255 Patientinnen [57] zeigten dabei gute Ergebnisse für die internetbasierte KVT-I, sowohl in Bezug auf insomnische Symptome als auch in Bezug auf Fatigue. $\mathrm{Zu}$ dem liegt eine unkontrollierte Pilotstudie zur internetbasierten KVT-I bei Patienten mit Asthma vor, die ebenfalls eine gute Effektivität für die Reduktion sowohl insomnie- als auch asthmaspezifischer Beschwerden zeigte [34]. Die Teilnahme an einer internetbasierten KVT-I wirkte sich in randomisierten klinischen Studien außerdem auch positiv auf insomnische Beschwerden von Patienten mit Bluthochdruck [35] und Schmerzstörungen [46] aus.

\section{Internetbasierte KVT-I bei Kindern, Jugendlichen und jungen Erwachsenen}

Die internetbasierte KVT-I wurde außerdem bei Kindern und Jugendlichen eingesetzt und auf ihre Effektivität hin überprüft. So zeigten Schlarb et al. [45] in einer unkontrollierten Studie Verbesserungen nach einer auf kognitiv-verhaltenstherapeutischen Prinzipien beruhenden Behandlung für Kinder im Alter von 6 Monaten bis 4 Jahren. Mindell et al. [36] zeigten ebenfalls eine gute Wirksamkeit eines internetbasierten KVT-I-
Programms für Kinder hinsichtlich der Einschlaflatenz und der Anzahl und Dauer nächtlicher Wachphasen. Zudem legten Werner-Seidler et al. [54] eine Übersichtsarbeit zur internetbasierten KVT-I im Jugendalter und frühen Erwachsenenalter vor. Dabei zeigten sich in bislang drei Originalstudien ähnlich gute Effekte wie durch die internetbasierte KVT-I bei Erwachsenen.

\section{Untersuchungen zum Therapieprozess}

Im Vergleich zu der beträchtlichen Anzahl an Studien zur generellen Effektivität gibt es bislang wenige Untersuchungen zur optimalen konkreten Ausgestaltung der internetbasierten Interventionen bei Insomnie. Bei anderen psychischen Störungen wurde diesbezüglich am häufigsten untersucht, ob die Effektivität einer internetbasierten Behandlung gesteigert werden kann, indem ein Kontakt zu Therapeuten per E-Mail, Video oder Telefon in die Behandlung integriert wird. Dabei zeigte sich eine signifikante Überlegenheit therapeutisch begleiteter gegenüber therapeutisch unbegleiteten internetbasierten Interventionen [2]. Im Kontext der internetbasierten KVT-I für Erwachsene liegt zu dieser Fragestellung bislang nur eine empirische Studie vor [31]. In dieser wurden 262 Patienten randomisiert einer internetbasierten KVT-I mit bzw. ohne E-Mail-Support zugewiesen, wobei die Gruppe, die wöchentliche motivierende E-Mails erhielt, sowohl direkt nach Therapieende als auch im 6-MonatsFollow-Up signifikant besser abschnitt als die Gruppe ohne Support. Die Studie legt also nahe, dass ein persönlicher Kontakt via E-Mail durchaus hilfreich sein kann, um die Effektivität einer internetbasierten Behandlung zu steigern. Auch für das Kindesalter wurde der zusätzliche Effekt durch solche Interventionsbedingungen überprüft. So wurden in der Behandlung von jungen Kindern mit Insomnie 199 Eltern mit ihren Kindern randomisiert einer KVT-I Bedingung, der KVT-I mit wöchentlichem Telefonsupport oder einer Kontrollbedingung (Warteliste) zugewiesen. Bei beiden Interventionsbedingungen ergaben sich signifikante Verbesserungen. Besonders 
die Drop-out-Rate konnte durch die telefonische Unterstützung verringert werden [44]. Bemerkenswert ist aus der Forschung zu anderen psychischen Störungen, dass auch zu virtuellen Therapeuten eine Psychotherapiebeziehung aufgebaut werden kann [30], sodass weitere Untersuchungen $\mathrm{zu}$ diesem Themenkomplex unabdingbar erscheinen.

Eine weitere kürzlich erschienene Arbeit zur optimalen Ausgestaltung der internetbasierten Behandlung der Insomnie zeigt, dass es günstig ist, potenzielle Non-Responder auf die Behandlung frühzeitig $\mathrm{zu}$ erkennen und einer intensiveren Behandlung zuzuführen [23]. In dieser Arbeit erhielten 251 erwachsene Patienten mit Insomnie eine internetbasierte KVT-I mit Therapeutenunterstützung. Bei einer Teilgruppe von 102 Patienten wurde bereits frühzeitig im therapeutischen Prozess ein erhöhtes Risiko für eine Non-Response festgestellt. Diese Teilgruppe wurde daraufhin randomisiert einer intensivierten Behandlung mit mehr Therapeutenunterstützung oder der Standardbehandlung zugewiesen, wobei die intensivierte Behandlung in Bezug auf die Effektivität signifikant besser abschnitt.

Darüber hinaus legen qualitative Arbeiten nahe, dass es wichtig ist, die individuellen Bedürfnisse von Patienten zu berücksichtigen, wenn es um die Entscheidung geht, ob eine internetbasierte oder F2F-KVT-I empfohlen wird [13], und dass insbesondere die Bettzeitrestriktion im internetbasierten Format teils als problematisch empfunden wird [11]. Zusammengefasst erscheinen aber weitere Forschungsarbeiten erforderlich zu sein, um abschließende Aussagen zur optimalen Ausgestaltung der internetbasierten Behandlung von Insomnien treffen zu können.

\section{Publikationen aus dem deutschsprachigen Raum}

Auch wenn verschiedene Anbieter im deutschsprachigen Raum internetbasierte Therapieangebote für Patienten mit Insomnien zur Verfügung stellen, u. a. auch verschiedene Krankenkassen in Deutschland (z. B. BARMER, DAKGesundheit, Techniker Krankenkasse), gibt es bislang vergleichsweise wenige wissenschaftliche Untersuchungen zur Effektivität dieser Behandlungen. So liegen bislang drei Publikationen aus einer Arbeitsgruppe aus Lüneburg und Erlangen in Zusammenarbeit mit dem GET.ON Institut [17, 49, 50], eine Publikation aus der Schweiz [33] sowie die oben bereits beschriebenen Arbeiten zur Behandlung von Schlafstörungen bei Kleinkindern [44, 45] vor.

Die internetbasierte KVT-I des GET. ON Instituts zeigte dabei eine gute Effektivität spezifisch bei Arbeitnehmern mit beruflichem Stress. Es wurden hierbei zwei randomisierte klinische Studien mit jeweils 128 Studienteilnehmern durchgeführt, die entweder der internetbasierten KVT-I oder einer Wartelistenkontrollbedingung zugewiesen wurden $[17,49]$. Die Datenauswertung von Thiart et al. [50] beinhaltete dabei auch eine gesundheitsökonomische Analyse, die zeigte, dass die Intervention kosteneffektiv ist, insbesondere durch die Effekte auf Präsentismus. Präsentismus, also die durch Krankheit bedingte Reduktion der Produktivität am Arbeitsplatz bei Anwesenheit am Arbeitsplatz, konnte durch die Intervention deutlich reduziert werden und somit auch die indirekten Kosten der Schlafstörung [50].

Darüber hinaus haben Lorenz et al. [33] die Effektivität einer internetbasierten Behandlung für Insomnie gegenüber einer Wartelistenkontrollbedingung in einer randomisierten klinischen Studie untersucht. Dabei wurden 55 erwachsene Patienten randomisiert der internetbasierten Behandlung „mementor somnium“" oder einer Wartelistenkontrollbedingung zugewiesen. Bei der internetbasierten Behandlung handelt es sich um ein vollautomatisiertes Programm ohne Therapeutenunterstützung mit einer Behandlungsdauer von 6 Sitzungen von jeweils ungefähr $30 \mathrm{~min}$. Die Analyse der Forschungsdaten zeigte große Effektstärken für die Schwere der insomnischen Symptomatik, zudem zeigte eine Follow-up-Untersuchung nach 12 Monaten eine gute Stabilität der erzielten Ergebnisse.

Weiterhin ist anzumerken, dass in der klinischen Routineversorgung in der Schweiz seit dem 01.01.2017 eine internetbasierte KVT-I zu Lasten der Grundversicherung landesweit verfügbar ist (KSM-SOMNET, maximale Therapiedauer 16 Wochen). Patienten mit relevanter psychiatrischer Komorbidität werden dabei als potenzielle Non-Responder einem F2F-Therapeutenkontakt zugeführt.

\section{Diskussion}

Die internetbasierte KVT-I ist eine derzeit sehr intensiv beforschte psychologische Intervention, was sich unter anderem daran zeigt, dass die meisten zitierten Arbeiten aus den letzten Jahren stammen. Die Daten zur Wirksamkeit sind durchgehend sehr überzeugend, sowohl in Bezug auf schlafbezogene Variablen als auch in Bezug auf Depressivität, Angst, Fatigue und Lebensqualität. Bezüglich der Wirksamkeit der internetbasierten KVT-I bei erwachsenen Patienten mit komorbiden psychischen Störungen oder körperlichen Erkrankungen erscheint die Evidenz aktuell noch etwas uneinheitlich bzw. vorläufig zu sein, sodass weitere Forschung auf diesem Gebiet erforderlich ist. Dies gilt ebenso für die wichtige Frage, inwiefern es die Effektivität steigert, wenn ein persönlicher Kontakt zu Therapeuten per E-Mail, Video oder Telefon in die Behandlung integriert wird.

\section{Stichprobenzusammensetzung}

Auffällig ist, dass in einigen Studien zur internetbasierten KVT-I deutlich größere Stichproben untersucht wurden (mit zum Teil über 1000 Versuchspersonen pro Studie) als in den entsprechenden F2F-Studien zur KVT-I. Während dies einerseits große Vorteile in Bezug auf die statistische Validität der Daten mit sich bringt, ist auch zu beachten, dass das Screening von Versuchspersonen in Studien zur internetbasierten KVT-I zum Teil weniger umfassend erfolgt als in entsprechenden Studien zur F2F-Behandlung. Insbesondere gibt es Arbeiten, in denen es nur telefonischen Kontakt (z.B. Ritterband et al. [42]) oder überhaupt keinen persönlichen Kontakt (z. B. Espie et al. [22]) zwischen Forschungsteams und Versuchspersonen gab und 
auch das Screening vollständig per Telefon bzw. online erfolgte. Das hat zur Folge, dass nicht alle Studien ärztlich oder psychotherapeutisch gestellte Insomnie-Diagnosen laut ICD-10 oder DSM-5 verwenden, sondern teilweise nur Insomniesymptome vorliegen. Auch liegt die Vermutung nahe, dass Studien mit Untersuchungen im Schlaflabor eher Patienten mit schwereren Problemen ansprechen. Damit verbundene Unterschiede in der Stichprobenzusammensetzung zwischen F2F-Studien und Studien zur internetbasierten KVT-I sollten daher näher untersucht werden.

\section{Datenerhebung}

Die angesprochene Thematik wirft die Frage der Diagnostik der Insomnie in den entsprechenden Studien auf. Zur Validierung von Fragebögen für den digitalen Gebrauch, die ursprünglich als Papierund-Bleistift-Fragebogen validiert wurden, gibt es insgesamt eine sehr gute Vergleichbarkeit der Daten aus den beiden Erhebungskontexten [29, 51], auch wenn formal bemängelt werden kann, dass nicht alle in den entsprechenden Studien eingesetzten Fragebögen auch für den Gebrauch im Internet validiert wurden. Es gibt im Insomniebereich darüber hinaus inzwischen auch Fragebögen, die spezifisch für den digitalen $\mathrm{Ge}$ brauch validiert wurden [21]. So ist auf dem digitalen Weg auch eine Datenerhebung für ätiologische Studien möglich, was aufgrund der leichteren Rekrutierung von großen Stichprobenumfängen in den letzten Jahren auch im Insomniebereich zugenommen hat $[3,5]$. Jedoch sollte auch hier kritisch die Schwere der Störung geprüft werden. In vielen Studien werden Insomniesymptome adressiert und nicht unbedingt chronifizierte klinisch diagnostizierte Insomnien.

\section{Nutzungsverhalten und weitere Variablen}

Grundsätzlich ist in Bezug auf die zunehmende Untersuchung von internetbasierter KVT-I kritisch anzumerken, dass es Hinweise gibt, dass eine intensive Nutzung digitaler Medien mit Schlafstörungen assoziiert ist [4], insbesondere wenn diese Nutzung abends im Bett erfolgt [24]. Daher scheint eine intensivere Auseinandersetzung mit der Frage, zu welchen Uhrzeiten Patienten bevorzugt digitale Behandlungsangebote nutzen sollten, sinnvoll.

Kritisch muss auch diskutiert werden, inwieweit der Phänotyp einer Insomnie Einfluss auf die Effektivität der internetbasierten KVT-I hat. Variablen, die hierbei möglicherweise relevant sind, sind das Alter der Versuchspersonen, die Dauer der bestehenden Insomnie, Art und Umfang eventueller medikamentöser oder psychotherapeutischer Vorbehandlungen, Komorbiditäten oder spezifische Subtypen der Insomnie.

Insgesamt sind die Daten zur Wirksamkeit der digitalen KVT-I überzeugend, es bleiben jedoch einige relevante Forschungsfragen zu beantworten, bevor die internet- und mobil-basierte KVT-I flächendeckend implementiert werden kann. Dazu zählen unter anderem: 1) die Frage der Wirksamkeit unter Routinebedingungen im Allgemeinen sowie bei technikaffinen und bildungsnahen $\mathrm{Pa}$ tienten im Spezifischen; 2) die Frage nach der optimalen Intensität der therapeutischen Begleitung der internet- und mobil-basierten KVT-I unter den Gesichtspunkten Wirksamkeit und KostenEffektivität; 3) die Frage, für welche Patientengruppen (komorbide psychische und/oder körperliche Erkrankungen vs. keine Komorbidität) welches Vorgehen indiziert ist; und 4) ob es spezifische unerwünschte Nebenwirkungen der internetbasierten KVT-I gibt, bzw. ob die behavioralen Techniken (Bettzeitrestriktion, Stimuluskontrolle) ohne therapeutische Begleitung auch problematisch sein können.

\section{Korrespondenzadresse}

Prof. Dr. Dr. Kai Spiegelhalder

Klinik für Psychiatrie und Psychotherapie, Universitätsklinikum Freiburg, Medizinische Fakultät, Albert-Ludwigs-Universität Freiburg Hauptstr. 5, 79104 Freiburg, Deutschland kai.spiegelhalder@uniklinik-freiburg.de

Funding. Open Access funding provided by Projekt DEAL.

\section{Einhaltung ethischer Richtlinien}

Interessenkonflikt. K. Spiegelhalder, J. Acker, H. Baumeister, A. Büttner-Teleaga, H. Danker-Hopfe, D.D. Ebert, I. Fietze, L. Frase, D. Lehr, A. Maun, I. Mertel, K. Richter, D. Riemann, C. Sauter, C. Schilling, A.A. Schlarb, M. Specht, L. Steinmetz, H.G. Weeß und T. Crönlein geben an, dass kein Interessenkonflikt besteht. S. Klein ist Mitarbeiterin der BARMER.

Für diesen Beitrag wurden von den Autoren keine Studien an Menschen oder Tieren durchgeführt. Für die aufgeführten Studien gelten die jeweils dort angegebenen ethischen Richtlinien.

Open Access. Dieser Artikel wird unter der Creative Commons Namensnennung 4.0 International Lizenz veröffentlicht, welche die Nutzung, Vervielfältigung, Bearbeitung, Verbreitung und Wiedergabe in jeglichem Medium und Format erlaubt, sofern Sie den/die ursprünglichen Autor(en) und die Quelle ordnungsgemäß nennen, einen Link zur Creative Commons Lizenz beifügen und angeben, ob Änderungen vorgenommen wurden.

Die in diesem Artikel enthaltenen Bilder und sonstiges Drittmaterial unterliegen ebenfalls der genannten Creative Commons Lizenz, sofern sich aus der Abbildungslegende nichts anderes ergibt. Sofern das betreffende Material nicht unter der genannten Creative Commons Lizenz steht und die betreffende Handlung nicht nach gesetzlichen Vorschriften erlaubt ist, ist für die oben aufgeführten Weiterverwendungen des Materials die Einwilligung des jeweiligen Rechteinhabers einzuholen.

Weitere Details zur Lizenz entnehmen Sie bitte der Lizenzinformation auf http://creativecommons.org/ licenses/by/4.0/deed.de.

\section{Literatur}

1. American Psychiatric Association (2013) Diagnostic and statistical manual of mental disorders, 5. Aufl. American Psychiatric Publishing, Washington, D.C.

2. Baumeister $H$, Reichler L, Munzinger $M$, Lin J (2014) The impact of guidance on Internet-based mental health interventions-a systematic review. Internet Interv 1(4):205-215

3. Benjamins JS, Migliorati F, Dekker K, Wassing R, Moens S, Blanken TF, Te Lindert BHW, Sjauw Mook J, Van Someren EJW (2017) Insomnia heterogeneity: characteristics to consider for datadriven multivariate subtyping. Sleep Med Rev 36:71-81

4. Bhat S, Pinto-Zipp G, Upadhyay H, Polos PG (2018) "To sleep, perchance to tweet": in-bed electronic social media use and its associations with insomnia, daytime sleepiness, mood, and sleep duration in adults. Sleep Health 4(2):166-173

5. Blanken TF, Benjamins JS, Borsboom D, Vermunt JK, Paquola C, Ramautar J, Dekker K, Stoffers D, Wassing R, Wei Y, Van Someren EJW (2019) Insomnia disorder subtypes derived from life history and traits of affect and personality. Lancet Psychiatry 6(2):151-163

6. Blom K, Jernelov S, Kraepelien M, Bergdahl MO, Jungmarker K, Ankartjarn L, Lindefors N, Kaldo V (2015) Internet treatment addressing either 
insomnia or depression, for patients with both diagnoses: a randomized trial.Sleep 38(2):267-277

7. Blom K, Tarkian Tillgren $H$, Wiklund T, Danlycke $E$, Forssen $M$, Soderstrom A, Johansson R, Hesser $H$, Jernelov S, Lindefors N, Andersson G, Kaldo V (2015) Internet-vs. group-delivered cognitive behavior therapy for insomnia: a randomized controlled non-inferiority trial. Behav Res Ther 70:47-55

8. Blom K, Jernelov S, Ruck C, Lindefors N, Kaldo V (2016) Three-year follow-up of insomnia and hypnotics after controlled Internet treatment for insomnia. Sleep 39(6):1267-1274

9. Blom K, Jernelov S, Ruck C, Lindefors N, Kaldo V (2017) Three-year follow-up comparing cognitive behavioral therapy for depression to cognitive behavioral therapy for insomnia, for patients with both diagnoses. Sleep. https://doi.org/10.1093/ sleep/zsx 108

10. Carlbring P, Andersson G, Cuijpers P, Riper $H$, Hedman-LagerlofE (2018) Internet-based vs. faceto-face cognitive behavior therapy for psychiatric and somatic disorders: an updated systematic review and meta-analysis. Cogn Behav Ther 47:1-18

11. Chan C, West S, Glozier N (2017) Commencing and persisting with a web-based cognitive behavioral intervention for insomnia: a qualitative study of treatment completers. J Med Internet Res 19(2):e37

12. Cheng $P$, Luik Al, Fellman-Couture $C$, Peterson $E$, Joseph CLM, Tallent G, Tran KM, Ahmedani BK, Roehrs T, Roth T, Drake CL (2019) Efficacy of digital CBT for insomnia to reduce depression across demographic groups: a randomized trial. Psychol Med 49(3):491-500

13. Cheung JMY, Bartlett DJ, Armour CL, Laba T-L, Saini B (2019) Patient perceptions of treatment delivery platforms for cognitive behavioral therapy for insomnia. Behav Sleep Med 17(1):81-97

14. Christensen H, Batterham PJ, Gosling JA, Ritterband LM, Griffiths KM, Thorndike FP, Glozier N, O'Dea B, Hickie IB, Mackinnon AJ (2016) Effectiveness of an online insomnia program (SHUti) for prevention of depressive episodes (the goodnight study): a randomised controlled trial. Lancet Psychiatry 3(4):333-341

15. Diem SJ, Ewing SK, Stone KL, Ancoli-Israel S, Redline S, Ensrud KE (2014) Use of non-benzodiazepine sedative hypnotics and risk of falls in older men. J Gerontol Geriatr Res 3(3):158

16. Dozeman E, Verdonck-de Leeuw IM, Savard J, van Straten A (2017) Guided web-based intervention for insomnia targeting breast cancer patients: feasibility and effect. Internet Interv 9:1-6

17. EbertDD, Berking M, Thiart H, Riper H, Laferton JAC, Cuijpers P, Sieland B, LehrD (2015) Restoring depleted resources: efficacy and mechanisms of change of an internet-based unguided recovery training for better sleep and psychological detachment from work. Health Psychol 34:1240-1251

18. Ebert DD, Van Daele T, Nordgreen T, Karekla $M_{\text {, }}$ Compare A, Zarbo C, Brugnera A, Overland $S$, Trebbi G, Jensen KL, Kaehlke F, Baumeister $\mathrm{H}$, Taylor J (2018) Internet and mobile-based psychological interventions: applications, efficacy and potential for improving mental health. A report of the EFPA E-health taskforce. Eur Psychol 23(3):167-187. https://doi.org/10.1027/10169040/a000318

19. Espie CA (2009) "Stepped care": a health technology solution for delivering cognitive behavioral therapy as a first line insomnia treatment. Sleep 32(12):1549-1558
20. Espie CA, Kyle SD, Williams C, Ong JC, Douglas NJ Hames P, Brown JSL (2012) A randomized, placebocontrolled trial of online cognitive behavioral therapy for chronic insomnia disorder delivered via an automated media-rich web application. Sleep 35(6):769-781

21. Espie CA, Farias Machado P, Carl JR, Kyle SD, Cape J, Siriwardena AN, Luik AI (2018) The sleep condition indicator: reference values derived from a sample of 200000 adults. J Sleep Res 27(3):e12643

22. Espie CA, Emsley R, Kyle SD, Gordon C, Drake CL, Siriwardena AN, Cape J, Ong JC, Sheaves B, Foster R, Freeman D, Costa-Font J, Marsden A, Luik AI (2019) Effect of digital cognitive behavioral therapy for insomnia on health, psychological well-being, and sleep-related quality of life: a randomized clinical trial. Jama Psychiatry 76(1):21-30

23. Forsell E, Jernelov S, Blom K, Kraepelien M, Svanborg C, Andersson G, Lindefors N, Kaldo V (2019) Proof of concept for an adaptive treatment strategy to prevent failures in Internet-delivered CBT: a single-blind randomized clinical trial with insomnia patients. Am JPsychiatry 176(4):315-323

24. Fossum IN, Nordnes LT, Storemark SS, Bjorvatn B, Pallesen $S$ (2014) The association between use of electronic media in bed before going to sleep and insomnia symptoms, daytime sleepiness, morningness, and chronotype. Behav Sleep Med 12(5):343-357

25. Glozier N, Christensen H, Griffiths KM, Hickie IB, Naismith SL, Biddle D, Overland S, Thorndike F, Ritterband L (2019) Adjunctive Internet-delivered cognitive behavioural therapy for insomnia in men with depression: a randomised controlled trial. Aust NZJ Psychiatry 53(4):350-360

26. Grobe TG, Steinmann S, Gerr J (2019) Gesundheitsreport 2019. Schriftenreihe zur Gesundheitsanalyse Bd. 17. BARMER, Berlin

27. Gosling JA, Batterham P, Ritterband L, Glozier N, Thorndike F, Griffiths KM, Mackinnon A, Christensen HM (2018) Online insomnia treatment and the reduction of anxiety symptoms as a secondary outcome in a randomised controlled trial: the role of cognitive-behavioural factors. Aust N Z J Psychiatry 52(12):1183-1193

28. Hagatun S, Vedaa O, Harvey AG, Nordgreen T, Smith ORF, Pallesen S, Havik OE, Thorndike FP, Ritterband LM, Sivertsen B (2018) Internet-delivered cognitive-behavioral therapy for insomnia and comorbid symptoms. Internet Interv 12:11-15

29. Hedman E, Ljotsson B, Blom K, El Alaoui S, Kraepelien M, Ruck C, Andersson G, Svanborg C, Lindefors N, Kaldo V (2013) Telephone versus internet administration of self-report measures of social anxiety, depressive symptoms, and insomnia: psychometric evaluation of a method to reduce the impact of missing data. J Med Internet Res 15(10):e229

30. Heim E, Rotger A, Lorenz N, Maercker A (2018) Working alliance with an avatar: how far can we go with internet interventions? Internet Interv 11:41-46

31. Lancee J, van den Bout J, Sorbi MJ, van Straten A (2013) Motivational support provided via email improves the effectiveness of internet-delivered self-help treatment for insomnia: a randomized trial. Behav Res Ther 51(12):797-805

32. Lancee J, van Straten A, Morina N, Kaldo V, Kamphuis JH (2016) Guided online or face-toface cognitive behavioral treatment for insomnia: a randomized wait-list controlled trial. Sleep 39(1):183-191

33. Lorenz N, Heim E, Roetger A, Birrer E, Maercker A (2019) Randomized controlled trial to test the efficacy of an unguided online intervention with automated feedback for the treatment of insomnia. Behav Cogn Psychother 47(3):287-302

34. Luyster FS, Ritterband LM, Sereika SM, Buysse DJ, Wenzel SE, Strollo PJ (2020) Internet-based cognitive-behavioral therapy for insomnia in adults with asthma: a pilot study. Behav Sleep Med 18(1):10-22. https://doi.org/10.1080/15402002. 2018.1518229

35. McGrath ER, Espie CA, Power A, Murphy AW, Newell J, Kelly C, Duffy N, Gunning P, Gibson I, Bostock S, O'Donnell MJ (2017) Sleep to lower elevated blood pressure: a randomized controlled trial (SLEPT). Am J Hypertens 30(3):319-327

36. Mindell JA, Du Mond CE, Sadeh A, Telofski LS, Kulkarni N, Gunn E (2011) Efficacy of an internetbased intervention for infant and toddler sleep disturbances. Sleep 34(4):451-458

37. Ohayon MM (2002) Epidemiology of insomnia: what we know and what we still need to learn. Sleep Med Rev 6:97-111

38. Qaseem A, Kansagara D, Forciea MA, Cooke M, Denberg TD (2016) Management of chronic insomnia disorder in adults: a clinical practice guideline from the American college of physicians. Ann Intern Med 165(2):125-133

39. Riemann D, Baglioni C, Bassetti C, Bjorvatn B, Dolenc Groselj L, Ellis JG, Espie CA, Garcia-Borreguero D, Gjerstad M, Goncalves M, Hertenstein $E_{t}$ Jansson-Frojmark $M$, Jennum PJ, Leger D, Nissen C Parrino L, Paunio T, Pevernagie D, Verbraecken J, Weess H-G, Wichniak A, Zavalko I, Arnardottir ES, Deleanu O-C, Strazisar B, Zoetmulder M, Spiegelhalder K (2017) European guideline for the diagnosis and treatment of insomnia. J Sleep Res 26(6):675-700

40. Riemann D, Baum E, Cohrs S, Crönlein T, Hajak G, Hertenstein E, Klose P, Langhorst J, Mayer G, Nissen C, Pollmächer T, Rabstein S, Schlarb A, Sitter H, Weeß HG, Wetter T, Spiegelhalder K (2017) S3Leitlinie Nicht erholsamer Schlaf/Schlafstörungen -Kapitel Insomnie bei Erwachsenen, Update 2016 Somnologie 21(1):2-44

41. Ritterband LM, Thorndike FP, Gonder-Frederick LA, Magee JC, Bailey ET, Saylor DK, Morin CM (2009) Efficacy of an Internet-based behavioral intervention for adults with insomnia. Arch Gen Psychiatry 66(7):692-698

42. Ritterband LM, Thorndike FP, Ingersoll KS, Lord HR, Gonder-Frederick L, Frederick C, Quigg MS Cohn WF, Morin CM (2017) Effect of a webbased cognitive behavior therapy for insomnia intervention with 1-year follow-up: a randomized clinical trial. JAMA Psychiatry 74(1):68-75

43. Schlack R, Hapke U, Maske U, Busch M, Cohrs S (2013) Frequency and distribution of sleep problems and insomnia in the adult population in Germany: results of the German health interview and examination survey for adults (DEGS1). Bundesgesundheitsblatt Gesundheitsforschung Gesundheitsschutz 56(5-6):740-748

44. Schlarb AA, Brandhorst I, Peters E, Hautzinger M (2017) Telephone support in an internet-based treatment for sleep problems in early childhood. J Psychiatry. https://doi.org/10.4172/2378-5756. 1000435

45. Schlarb AA, Brandhorst I (2012) Mini-KiSS Online: an internet-based intervention program for parents of young children with sleep problems-influence on parental behavior and children's sleep. Nat Sci Sleep 4:41-52

46. Shaffer KM, Camacho F, Lord HR, Chow PI, Palermo T, Law E, Thorndike FP, Ingersoll KS, Ritterband LM (2019) Do treatment effects of 
a web-based cognitive behavioral therapy for insomnia intervention differ for users with and without pain interference? A secondary data analysis. J Behav Med. https://doi.org/10.1007/ s10865-019-00065-w

47. Shochat T, Umphress J, Israel AG, Ancoli-Israel S (1999) Insomnia in primary care patients. Sleep 22:359-365

48. Taylor DJ, Peterson AL, Pruiksma KE, YoungMcCaughan S, Nicholson K, Mintz J (2017) Internet and in-person cognitive behavioral therapy for insomnia in military personnel: a randomized clinical trial. Sleep. https://doi.org/10.1093/sleep/ zsx075

49. ThiartH, LehrD, EbertDD, Berking M, RiperH(2015) Log in and breathe out: internet-based recovery training for sleepless employees with work-related strain-results of a randomized controlled trial. Scand JWork Environ Health 41(2):164-174

50. Thiart H, Ebert DD, Lehr D, Nobis S, Buntrock $C$, Berking M, Smit F, Riper H (2016) Internet-based cognitive behavioral therapy for insomnia:a health economic evaluation. Sleep 39(10):1769-1778

51. Thorndike FP, Ritterband LM, Saylor DK, Magee JC, Gonder-Frederick LA, Morin CM (2011) Validation of the insomnia severity index as a web-based measure. Behav Sleep Med 9(4):216-223

52. van Straten $A$, van der Zweerde T, Kleiboer $A$, Cuijpers P, Morin CM, Lancee J (2018) Cognitive and behavioral therapies in the treatment of insomnia: a meta-analysis. Sleep Med Rev 38:3-16

53. Vedaa $\mathrm{O}$, Hagatun $\mathrm{S}$, Kallestad $\mathrm{H}$, Pallesen $\mathrm{S}$, Smith ORF, Thorndike FP, Ritterband LM, Sivertsen B (2019) Long-term effects of an unguided online cognitive behavioral therapy for chronic insomnia. JClin Sleep Med 15(1):101-110

54. Werner-Seidler A, Johnston L, Christensen H (2018) Digitally-delivered cognitive-behavioural therapy for youth insomnia: a systematic review. Internet Interv 11:71-78

55. World Health Organization (1992) International statistical classification of diseases and related health problems (ICD-10). Huber, Bern.

56. Zachariae R, Lyby MS, Ritterband LM, O'Toole MS (2016) Efficacy of internet-delivered cognitive-behavioral therapy for insomnia-a systematic review and meta-analysis of randomized controlled trials. Sleep Med Rev 30:1-10

57. Zachariae R, Amidi A, Damholdt MF, Clausen CDR, Dahlgaard J, Lord H, Thorndike FP, Ritterband LM (2018) Internet-delivered cognitive-behavioral therapy for insomnia in breast cancer survivors: a randomized controlled trial. J Natl Cancer Inst 110(8):880-887

\section{J. Niemann \\ Patient Krankenhaus - Doktor Faber hat Dienst}

Ein gelungener "Snapshot" der Krankenhausrealität

Lüchow: Jeetzelbuch 2018, 1. Auflage, 267 S., (ISBN: 978-3-928117-34-0), Kartoniert 16,80 EUR

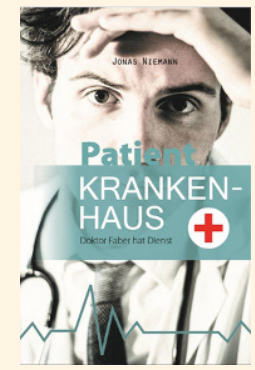

„Patient Krankenhaus - Doktor Faber

hat Dienst", ist im Jeetzelbuch-Verlag erschienen und wurde von Jonas Niemann geschrieben. Wie einer der Protagonisten des Buches begrüßte auch ich am Tag nach der Lektüre meinen Kollegen überschwänglich mit den Worten: „Gefechtsbereitschaft Korken!" Als Krankenhausarzt erkennt man sich in dem Roman sofort wieder. Auch meine Frau, selbst keine Ärztin, konnte sich mit dem Buch identifizieren.

Die Geschichte von Doktor Faber ist authentisch und glaubwürdig geschrieben, das macht sie persönlich und besonders interessant. Das Buchende kam unerwartet schnell: „Waaas? Schon fertig? Ich will mehr! " „Patient Krankenhaus - Doktor Faber hat Dienst" ist kein dahin phantasierter Kunstroman, auch keine Dokumentation. Es ist vielmehr eine realistisch und spannend geschriebene Reportage und gleichzeitig eine Hommage an die Krankenhäuser der Vergangenheit. Warum der Vergangenheit? Weil mit der Gesundheitsreform der rotgrünen Koalition von 2004 das Gesundheitssystem weiter umgebaut wurde, weg von einem System auf Solidaritätsbasis hin zu einem neoliberalen, "marktgeregelten" und gewinnorientierten Kundenservice. Eine Patientenklingel drücken, und sofort kommt eine Krankenschwester: Das waren noch schöne Zeiten! Dieser Moment, in dem in deinem Arztkittel gleichzeitig drei Telefone klingeln: Wie soll man sich dabei fühlen? Wichtig oder ausgebeutet?
Die Überlegungen des Autors über Empathie, das Leben und den Tod, über den Verlust von Taktgefühl, über Patienten- und auch Arztautonomie, über die Folgen des ökonomischen Drucks im Krankenhaus, und seine Etüden über Patienten, Pfleger, Ärzte - all' das schmerzt und amüsiert zugleich und zwingt den Leser zu reflektieren und nachzudenken.

Dem Autor ist es gelungen einen "snapshot" der Krankenhausrealität zu machen, ohne es künstlich mit Brutalität, Skandalen, Sex und übertriebenen Gefühlen zu füllen. Es wäre dann ein ganz anderes Buch geworden. Ich mag das Buch so, wie es ist, weil der beschriebene Krankenhausalltag auch mein Leben war.

Ein sehr schönes Gefühl!

Danke Jonas Niemann. 